\title{
Applications of Novel Blood Tests of Osteosarcoma Biomarkers for Diagnosis and Prognosis in Pediatric Patients
}

\author{
Yulia A. Savitskaya ${ }^{1}$ \\ ${ }^{1}$ Tissue Engineering, Cell Therapy and Regenerative Medicine Unit, National Institute of Rehabilitation, \\ Mexico`s Ministry of Health, Mexico City, Mexico \\ Correspondence: Yulia Anatolevna Savitskaya, Tissue Engineering, Cell Therapy and Regenerative Medicine \\ Unit, National Institute of Rehabilitation, Mexico`s Ministry of Health, Calzada Mexico-Xochimilco No289, \\ Colonia Arenal de Guadalupe, Delegación Tlalpan, Mexico, Distrito Federal, Mexico, Codigo Postal 14389. Tel: \\ 52-55-5999-1000 Ext 18333/19604. E-mail: yulia.savitskaya2012@gmail.com
}

Received: October 30, 2012 Accepted: November 18, 2012 Online Published: December 11, 2012

doi:10.5539/cco.v2n1p28 URL: http://dx.doi.org/10.5539/cco.v2n1p28

The author declares no competing interests

This work was supported by a grant from Foundation FONSEC SSA/IMSS/ISSSTE-CONACyT (number SALUD-2010-01-138883 to Y.A.S.)

Abbreviations: ANG-IgM, natural IgM antibodies to angiogenin; ANG, angiogenin; CTA, computed tomography angiography; ELISA, enzyme-linked immunosorbent assay; fT3, free triiodothyronine; IgM, immunoglobulin M; OS, osteosarcoma; POP, pediatric osteosarcoma patient; VEGF, vascular endothelial growth factor.

\begin{abstract}
Background: Osteosarcoma is the most frequent malignant bone tumor in pediatric patients occurring in the second decade of life between ages 10 and 15 . An understanding of the balance between innate immunity, tumor angiogenesis and thyroid hormone status may assist in pediatric osteosarcoma diagnostics and therapeutics. Serum tumor markers open up new prospects for early diagnosis and treatment of pediatric osteosarcoma.

Objectives: The aim of this work was to investigate the possible role of natural IgM antibodies to angiogenin (ANG-IgM) as an early biomolecule involved in the progression of pediatric osteosarcoma and identify the combination of serum markers differentiating between osteosarcoma patients and healthy individuals.
\end{abstract}

Methods: We studied 50 pediatric patients newly diagnosed with osteosarcoma (average age 14.5 years). The comparative group consisted of 50 patients with other types of pediatric bone tumors matched for age, gender and ethnicity. In addition, a healthy control group including 50 age- and sex-matched children was included in the study. Serum ANG-IgM levels were determined by novel ELISA technique (INR, Mexico City, Mexico). Serum fT3 levels were measured using a commercial ELISA kit (Diagnostic Automation, INC. CA, USA) according to the manufacturer's protocols.

Results: The level of ANG-IgM was higher in all types of bone tumors compared to normal healthy children. No significant difference was detected in the level of ANG-IgM among boys and girls. Statistically significant difference was found across the different types of bone tumors. Significant correlation was observed between serum levels of ANG-IgM and fT3 in children.

Conclusions: Combinations of ANG-IgM and fT3 may be administered together. The potential effect of an intrinsic dynamic balance of tumor angiogenesis residing within a single hormone (fT3) is an attractive concept for regulation of osteosarcoma vascularization.

Keywords: pediatric osteosarcoma, serum tumor markers, enzyme-linked immunosorbent assay, tumor angiogenesis, innate immunity, thyroid hormone homeostasis 


\section{Background}

Cancer is a major public health problem in all parts of the world (Siegel, 2012). Recent statistical data indicate a steady and intensive increase of mortality and morbidity in bone tumors (Ottaviani, 2009). Osteosarcoma makes up to 50\%-70\% of all observed cases of skeletal system tumors (Picci, 2007). Osteosarcoma is a malignant tumor in which newly developed bone or osteoid is produced by the tumor cells (Wang, 2005; Fuchs 2002). Statistics (Mirbello, 2009) suggest that osteosarcoma is the most commonly found pediatric bone tumor and is seventh most common malignant pediatric bone tumor (Estrada-Villaseñor, 2008). This tumor commonly occurs in children in the second decade of life, between ages 10 and 15 (Tunn, 2004), although it also may affect children younger than 5 years old (Rendon-Macias, 2005).

The incidence of osteosarcoma in Mexico approaches 300 cases per year, with the majority of cases occurring between 2 and 20 years of age (IMSS, 2005; Gomez-Dantes, 2011). Osteosarcoma patients are getting younger, with an increasing number of youths and even children being diagnosed (Orellana, 2005). In Mexico, 74\% of the identified bone tumor cases in youths and children were due to osteosarcoma (Luna-Ortiz, 2010).

Modern medicine has shown great strides in treating pediatric osteosarcoma (Benjamin, 2009). Osteosarcoma is treatable, although only at the early stages (Marina, 2004). While early diagnosis of osteosarcoma is complicated to a high degree, lately there have appeared a possibility of timely detection of osteosarcoma through a specialized tumor marker analysis (Adrianov, 2008; DuBois, 2007; Kushlinskii, 2000; Pepe, 2001). The particularities of osteosarcoma and the specifics of its "biological behavior" are known to be characterized by tissue, cell or molecular markers. These markers describe fundamental osteosarcoma cell qualities such as metastases, invasion, unchecked proliferation, neoangiogenetic activity, ability to inhibit apoptosis, and cell sensibility to exogenous and endogenous regulators. Several new tumor markers are discovered every year, shedding light on the mechanisms of osteosarcoma progression in children (Gelberg, 1997; Pierz, 2001). The pathogenesis of pediatric osteosarcoma is not quite clear (Broadhead, 2011; Ladanyi, 2000; Longhi, 2001). Laboratory diagnostics of pediatric osteosarcoma relies upon sensitive tumor-specific (i.e. associated with osteosarcoma) tumor markers in order to diagnose and monitor disease progression (Kaya, 2000). Of particular interest are the dynamics of the marker. Test specificity increases as multiple tumor markers are used together (Biomarkers Definitions Working Group, 2001; Cho-Chung, 2006). Part of the tumor markers is released into bloodstream, making it possible to determine their level using immunoenzyme analysis, a biochemical technique used in cancer immunology to detect specific molecules in a sample (Hanash, 2002; Master, 2005). Notably, serum is an essential sample for the therapeutic diagnosis and control and for the search of biological tumor markers (Meng, 2007).

The search for new specific and inexpensive tumor markers for pediatric osteosarcoma is ongoing, because early detection of osteosarcoma translates into thousands and even millions saved lives.

Pediatric oncology has concerned itself greatly with identifying biomarkers of early osteosarcoma recurrence, growth, and therapeutic response. Finding reliable biomarkers to simplify clinical diagnosis and differentiation between osteosarcoma and other bone tumors is important from prognostic viewpoint and to advance research into pathogenic mechanisms underlying a diversity of bone tumors. Evaluating natural IgM antibodies to tumor-associated antigens in the peripheral blood is now used for more accurate diagnosis of osteosarcoma. Previous reports that ANG-IgM may serve as a biomarker introduced the idea that the ANG-IgM content may be indicative of osteosarcoma (Savitskaya, 2010). But as a rule, the types of cancer that develop in children are different from those commonly found in adults.

Thyroid hormone plays a major role in the regulation of bone metabolism (Pepene, 2001). High-affinity triiodothyronine (T3) binding sites have been indetified in osteoblastic cells and various osteoblastic osteosarcoma cell lineages (Williams, 1994), and expression of thyroid hormone receptors in situ in human bone has been demonstrated (Dutkowsky, 1993). An understanding of the balance between natural immunity, tumor angiogenesis and thyroid hormone status may assist in osteosarcoma diagnostics and therapeutics for children.

The aim of this work is to investigate the possible role of ANG-IgM as an early biomolecule involved in the progression of pediatric osteosarcoma and identify the molecular group differentiating osteosarcoma patients from healthy individuals to find serum biomarkers.

\section{Materials and Methods}

\subsection{Serum Samples}

Blood samples were collected from 50 children hospitalized at the Bone Tumors Department in National Institute of Rehabilitation (Mexico City, Mexico) newly diagnosed with osteosarcoma (average age 14.5 years). Blood 
samples were collected prior to and following chemotherapy and radiotherapy. This therapy was proposed to children following treatment protocols available in our institutions. The comparative group included 50 children with other types of bone tumors matched for age, gender and ethnicity at the Bone Tumors Department in National Institute of Rehabilitation (Mexico City, Mexico). Samples from normal healthy children $(\mathrm{n}=50$, average age 14.9) were obtained from the Blood Bank of the National Institute of Rehabilitation (Mexico City, Mexico). The samples were primarily intended for routine biochemical tests at the Clinical Pathology Laboratory in the National Institute of Rehabilitation (Mexico City, Mexico). Serum was separated by centrifugation at 4000 $\mathrm{x}$ g for 10 minutes. Sera not used for routine biochemical tests were used for further investigations. The samples were stored at $-80^{\circ} \mathrm{C}$ until assayed.

\subsection{Determination of Serum ANG-IgM Level}

In a previous study novel immunoconjugates were designed, synthesized and then used to develop a rapid, specific and sensitive ELISA method to detect ANG-IgM directly in the peripheral venous blood sera of humans (Savitskaya, 2010). We have developed a solid-phase enzyme-linked immunosorbent assay (ELISA) system, in which ANG coupled to high-molecular weight matrix (polyphenylacrylate) was coated on microtiter plates. Human serum samples were incubated on the plates, after which bound ANG-IgM was detected with mouse anti-human IgM-HRP. The optimal sensitivity and specificity of the assay was $91 \%$ and $84 \%$. Serum ANG-IgM levels in a cohort of healthy donors differed by more than a hundred-fold, whereas the fluctuation of ANG-IgM level in individuals over time was small (coefficient of variation ranged between 6-25\%). We used an ELISA to compare serum ANG-IgM levels in patients with bone tumors and healthy controls according to protocols (Savitskaya, 2010). Tests were performed in duplicate. All measurement (patients and control subjects) were made on the same day and under the same experimental conditions.

\subsection{Determination of Serum Free Triiodothyronine Level}

Serum-free triiodothyronine (fT3) levels were determined by using commercially available ELISA kit designed to measure human fT3 in the serum (Diagnostic Automation, INC. CA, USA) according to the manufacturer's instructions.

\subsection{Vascular Imaging of Osteosarcoma by CTA}

CTA was performed on all of the pediatric osteosarcoma patients. Iopamidol-370 was administered via injection at a rate of $7 \mathrm{~mL} / \mathrm{s}$ into the celiac artery, or $4 \mathrm{~mL} / \mathrm{s}$ via the right, the left or the proper hepatic artery, depending on tumor location. Tumor vascularity was assessed by enhancement during the arterial phase of CTA. In brief, tumors that were markedly enhanced by CTA were assessed as very hypervascular lesions, those minimally to mildly enhanced by CTA were assessed as hypervascular, and those slightly enhanced or not enhanced by CTA were assessed as hypovascular.

\subsection{Ethical Approval}

All patients and healthy controls provided informed, written consent and the study was approved by the Ethics Committee of National Institute of Rehabilitation, Mexico City, Mexico.

\subsection{Statistics}

Data were processed using Microsoft Excel ${ }^{\circledR}$ and STATISTICA Version 8.0. Data are expressed as mean \pm standard deviation (SD) unless otherwise noted (Excel $\left.{ }^{\circledR}\right)$. Statistical significance of the measured data was determined using STATISTICA. Differences with $\mathrm{P}<0.05$ were considered significant and were determined by using one-way ANOVA test, which was applied for means comparison.

\section{Results}

\subsection{ANG-IgM in the Sera of Pediatric Osteosarcoma Patients and Healthy Children}

IgM antibodies, reacting with ANG tested, were present in the sera of pediatric osteosarcoma patients as well as in the sera of normal healthy children (Table 1). Serum ANG-IgM levels were significantly higher in all pediatric osteosarcoma patients $(100 \%)$ as compared to normal healthy children $(\mathrm{P}<0.005)$. Serum ANG-IgM levels were significantly higher in the group of pediatric osteosarcoma patients as compared with any other tumors $(\mathrm{P}<0.001)$. For the different types of pediatric tumors, no significant differences could be observed, as all of them showed significant differences from normal healthy children. 
Table 1. Evaluation of ANG-IgM in the sera of pediatric patients with bone tumors and healthy individuals

\begin{tabular}{lll}
\hline Bone tumors & Serum ANG-IgM, ODx1000 & P value \\
\hline Osteosarcoma & $825 \pm 265$ & 0.005 \\
Other malignant bone tumors & $625 \pm 118$ & 0.001 \\
Benign tumors & $511 \pm 91$ & 0.005 \\
Healthy individuals & $499 \pm 163$ & \\
\hline
\end{tabular}

By means of comparative data analysis we found statistically significant differences between ANG-IgM indicators in the osteosarcoma patients group and the group of patients with benign tumors $(\mathrm{P}<0.05)$ and the control group $(\mathrm{P}<0.05)$. The highest values of ANG-IgM were found in the group of osteosarcoma patients. The lowest values were found in the group of patients with benign tumors. The comparative analysis of ANG-IgM levels in the osteosarcoma group and benign tumors groups depending on age and sex revealed no statistically significant results.

\subsection{ANG-IgM expression in the Sera of Pediatric Osteosarcoma Patients with/without Pulmonary Metastasis}

The results of this investigation suggest an existing dependence between ANG-IgM expression and lung metastasis (Table 2). As prognostic indicators to the first lung metastases, ANG-IgM can be useful for pediatric osteosarcoma. Evaluation of ANG-IgM in sera of pediatric osteosarcoma patients could prove helpful in predicting patients at high risk for metastatic disease.

Table 2. The relationships between dynamic changes of serum ANG-IgM levels and pulmonary metastases in pediatric osteosarcoma patients

\begin{tabular}{lll}
\hline \multicolumn{3}{l}{ Serum ANG-IgM levels in pediatric osteosarcoma patients } \\
\hline Osteosarcoma without & Osteosarcoma with & Healthy \\
pulmonary metastases & pulmonary metastases & individuals \\
$828 \pm 258, \mathrm{P}<0.001$ & $957 \pm 212, \mathrm{P}<0.005$ & $499 \pm 160$ \\
\hline
\end{tabular}

High serum ANG-IgM levels significantly correlated with clinicopathologic features of pediatric osteosarcoma patients, such as metastasis-free time $(\mathrm{r}=0.75 ; \mathrm{P}<0.001)$ (Table 3$)$. High serum ANG-IgM levels were significantly associated with poor treatment response $(\mathrm{P}<0.001)$.

Table 3. Relationship between circulating ANG-IgM and pulmonary metastases in pediatric osteosarcoma patients

\begin{tabular}{lll}
\hline \multicolumn{3}{l}{ Serum ANG-IgM levels in pediatric osteosarcoma patients } \\
\hline Time to first lung pulmonary & Pediatric osteosarcoma & $\begin{array}{l}\text { Healthy } \\
\text { individuals }\end{array}$ \\
metastases from osteosarcoma & patients & \\
$>12$ months & $378 \pm 91, \mathrm{P}<0.005$ & $499 \pm 163$ \\
$<12$ months & $401 \pm 132, \mathrm{P}<0.001$ & \\
\hline
\end{tabular}

This work demonstrated that the expression of ANG-IgM in the serum can be attributed to the degree of malignization and, consequently, ANG-IgM levels can be helpful to differentiate the diagnosis. It thus stands to reason to extend the range of markers used to treat pediatric osteosarcoma by adding an ANG-IgM marker.

\section{3 fT3 in the Sera of Pediatric Osteosarcoma Patients}

From previous works it is known that the expression of angiogenesis stimulator (fT3) correlates with clinical pathological features in pediatric osteosarcoma patients (Petrosyan, 2003). In support of this conjecture we found that the fT3 blood level in 5 patients oscillated from 4.0 to $5.2 \mathrm{pmol} / \mathrm{mL}$. In other patients (7 individuals) fT3 levels ranged from 5.8 to $7.4 \mathrm{pmol} / \mathrm{mL}$. fT3 level in healthy children varies between 3.2 and $5.2 \mathrm{pmol} / \mathrm{mL}$. Over the course of observation of the patients (from 6 to 24 months) that underwent treatment we found that the group of children with normal (i.e. staying within normal physiological values) levels of fT3 had remained in remission. In contrast, children that underwent treatment with a higher fT3 level were found to show the generalization of tumor. 


\subsection{Association between the Presence of Circulating ANG-IgM and Expression of fT3 in Pediatric Osteosarcoma Patients}

We studied the relationship between fT3 expression and the circulating levels of ANG-IgM in the sera of pediatric osteosarcoma patients. Significant correlation was noted between serum levels of ANG-IgM and fT3 in pediatric osteosarcoma patients (Table 4). Combinations of fT3 with ANG-IgM may be administered together. The potential effect of an intrinsic dynamic balance of tumor angiogenesis residing within a single hormone is an attractive concept for regulation of osteosarcoma vascularization.

Table 4. Detection of expression levels of free triiodothyronine in serum of donors and pediatric patients with osteosarcoma

\begin{tabular}{lccc}
\hline \multicolumn{1}{c}{ Study group } & $\begin{array}{c}\text { Serum fT3, } \\
\text { pmol/mL }\end{array}$ & $\begin{array}{c}\text { Serum ANG-IgM, } \\
\text { ODX1000 }\end{array}$ & P value \\
\hline $\begin{array}{l}\text { Generalization of malignant } \\
\text { process over next 1-3 months }\end{array}$ & $5.8-7.4$ & $799 \pm 225$ & 0.001 \\
Remission over 6-24 months & $4.0-5.2$ & $535 \pm 75$ & 0.001 \\
Healthy individuals & $3.2-5.2$ & $499 \pm 163$ & \\
\hline
\end{tabular}

\section{Discussion}

A large number of studies conducted so far have revealed that there is no single molecule clearly defining the status of pediatric osteosarcoma (Gorlick, 2010; Sawyers, 2008). It is thus suggested that the combination of several biomarkers in one specific pattern can determine more accurately a specific pediatric osteosarcoma. This molecular footprint can improve our understanding of the clinical situation of a child.

Osteosarcoma is the most common bone tumor found in children and adolescents (the first peak is in the 10-14-year-old age group) during the so called "growth spurt" accompanied by the increased expression of the growth hormone, sexual steroid hormones and polypeptidic growth factors (Sidorenko, 2003; Silha, 2005). This suggests a close relationship between the adolescent growth spurt and osteosarcoma.

Over the last 10 years in the literature several tumor markers of pediatric osteosarcoma have been described (Yaw, 1999). Contemporary monitoring of pediatric osteosarcoma patients involves a study of serological tumor-associated markers. The main purpose of the markers is to evaluate the treatment efficacy and reveal remission at the preclinical stage.

Based on the conducted studies we developed a test system for the analysis of ANG-IgM expression (Savitskaya, 2010). The system helped to reveal statistically significant differences between osteosarcoma and other types of bone tumors based on the ANG-IgM expression level. It is worth noting that the utilization of the test system holds promise in the search for diagnostic and prognostic markers of pediatric osteosarcoma, as well as during analysis of oncological pathologies and the creation of genetic networks to describe the tumor.

Of certain value for the prognosis is the biochemical blood test, since higher levels of ANG-IgM, fT3 and ANG are most commonly observed in more aggressive course of pediatric osteosarcoma.

ANG expression in osteosarcoma is correlated with high microvascular density, metastatic spread and poor prognosis (Folkman, 2006). Evidence of increased angiogenesis in pre-malignant lesions may serve as a surrogate marker for tumor development (Raica, 2009; Carmeliet, 2011). Blood fT3 levels indicate a remission during 6 to 24 months after treatment or tumor generalization within 1 to 3 months (Petrosyan, 2003).

Serum ANG-IgM could serve as a useful and convenient marker for the detection and prognosis of pediatric osteosarcoma. When researching the particularities of ANG-IgM expression in pediatric osteosarcoma we found:

冈 No significant sex-related differences in ANG-IgM expression.

Increased expression of ANG-IgM marker in subgroups of deceased children and patients with metastasis of the tumor. Poor prognostic increase was observed in the 0-10 age subgroup.

\Impact of ANG-IgM expression on the length of uneventful survival (the higher was the level of ANG-IgM expression, the significantly shorter was the length of uneventful survival). 
Circulating ANG-IgM is important in pathogenicity, diagnosis, prevention and control of children's osteosarcoma. The results of the work are also in agreement with the previously reported findings that measuring angiogenesis regulators such as natural IgM antibodies, reacting with ANG, in serum of adult patients with osteosarcoma provided a new and sensitive marker for osteosarcoma progression, metastasis, and prognosis (Vollmers, 2007; Zhong, 2004).

Given that the bulk of osteosarcomas are heterogeneous, all three biomarkers (ANG-IgM, fT3, ANG) need to be determined. ANG-IgM is an early indicator of treatment efficacy and can indicate the length of the remission after chemotherapy. After remission, all three biomarkers need to be determined as a group every 4 to 6 weeks to detect tumor progression. In $60 \%$ of all cases, monitoring makes it possible to diagnose relapses and/or metastases several weeks prior to clinical manifestation.

The time before the clinical manifestation of the symptoms allows the attending physician to conduct a thorough diagnostic examination and provide necessary treatment in a timely fashion. The simultaneous detection of all three markers can indicate the presence of the endocrine component of the relapse and make use of differentiated approaches to the treatment of pediatric osteosarcoma. All three tumor markers need to be detected when monitoring treatment. If any of them exhibits a value of at least $150 \%$ over the initial value, the treatment regimen must be reconsidered, as this indicates the absence of effect.

Therefore, thanks to the progress in tumor immunology, biochemistry, molecular biology and biotechnology, the tools available to researchers and clinicians have been expanded to include biologically significant indicators that may help to improve the prognosis of early pediatric osteosarcoma and choose an adjuvant therapy in extended tumor growth.

\section{Conclusions}

This work opens up new perspectives for the discovery of serum markers of pediatric osteosarcoma helpful in diagnosis, prognosis or follow-up of this disease. Serum tumor markers seemed to be ideal for early diagnosis of pediatric osteosarcoma. The combination of diverse markers in a specific molecular pattern or footprint may be more precise in determining the pediatric osteosarcoma. I hope that this novel ANG-IgM-specific ELISA will be used in additional studies that will prove its clinical efficacy, not only for the early diagnosis of pediatric osteosarcoma, but also for prognosis and the implementation of preventive steps for this disease.

\section{References}

Andrianov, A. V., Morgun, A. V., Taranushenko, T. E., \& Salmina, A. B. (2008). Significance of progression markers for children's osteogenic sarcoma. Sib Oncol J, 5(29), 1-4. Russian.

Benjamin, R. S., \& Patel, S. R. (2009). Pediatric and adult osteosarcoma: comparisons and contrasts in presentation and therapy. Cancer Treat Res, 152, 355-63. http://dx.doi.org/10.1007/978-1-4419-0284-9_19

Biomarkers Definitions Working Group. (2001). Biomarkers and surrogate end-points: preferred definitions and conceptual framework. Clin Pharmacol Ther, 69, 89-95. http://dx.doi.org/10.1067/mcp.2001.113989

Broadhead, M. L., Clark, J. C., Myers, D. E., Dass, C. R., \& Choong, P. F. (2011). The molecular pathogenesis of osteosarcoma: a review. Sarcoma, 959248. Epub 2011 Apr 13.

Carmeliet, P., \& Jain, R. K. (2011). Molecular mechanisms and clinical applications of angiogénesis. Nature, 473(7347), 298-307. http://dx.doi.org/10.1038/nature10144

Cho-Chung, Y. S. (2006). Autoantibodies biomarker in the detection of cancer. Biochim Biophys Acta, 1762, 587-591. http://dx.doi.org/10.1016/j.bbadis.2006.04.001

DuBois, S., \& Demetri, G. (2007). Markers of angiogenesis and clinical features in patients with sarcoma. Cancer, 109(5), 813-819. http://dx.doi.org/10.1002/cncr.22455

Dutkowsky, J. P., Smith, R. A., Calandruccio, R. A., \& Carnesale, P. G. (1993). Effect of fetal thyroid hormone (RT3) on sarcoma cells in culture. J Orthop Res, 11(3), 379-385. http://dx.doi.org/10.1002/jor.1100110309

Estrada-Villaseñor, E., Delgado-Cedillo, E. A., \& Rico-Martínez, G. (2008). Frequency of bone neoplasms in children. Acta Ortop Mex, 22(4), 238-242. Spanish.

Estrada-Villaseñor, E., Delgado-Cedillo, E. A., \& Rico-Martínez, G. (2008). Prevalence of bone neoplasms in adolescents and young adults. Acta Ortop Mex, 22(5), 316-320. Spanish.

Estrada-Villaseñor, E., Flores-Carmona, J. F., Delgado-Cedillo, E. A., \& Rico-Martínez, G. (2008). Bone tumor frequency in adults and elderly. Acta Ortop Mex, 22(6), 356-360. Spanish. 
Folkman, J. (2006). Angiogenesis. Ann Rev Med, 57, 1-18. http://dx.doi.org/10.1146/annurev.med.57.121304.131306

Fuchs, B., \& Pritchard, D. J. (2002). Etiology of osteosarcoma. Clin Orthop, 397, 40-52. http://dx.doi.org/10.109 7/00003086-200204000-00007

Gelberg, K. H., Fitzgerald, E. F., Hwang, S., \& Dubrow, R. (1997). Growth and development and other risk factors for osteosarcoma in children and young adults. Int $J$ Epidemiol, 26(2), 272-278. http://dx.doi.org/10.1093/ije/26.2.272

Gómez Dantés, O., Sesma, S., Becerril, V. M., Knaul, F. M., Arreola, H., \& Frenk, J. (2011). The health system of Mexico. Review. Salud Publica Mex, 53(Suppl 2), s220-232.

Gorlick, R., \& Khanna, C. (2010). Osteosarcoma. J Bone Miner Research, 25(4), 683-691. http://dx.doi.org/10.1002/jbmr.77

Hanash, S. M., \& Celis, J. E. (2002). The Human Proteome Organization: a mission to advance proteome knowledge. Mol Cell Proteomics, 1, 413-414. http://dx.doi.org/10.1074/mcp.R200002-MCP200

Kaya, M., Wada, T., Akatsuka, T., Kawaguchi, S., Nagoya, S., Shindoh, M., ... Ishii, S. (2000). Vascular endothelial growth factor expression in untreated osteosarcoma is predictive of pulmonary metastasis and poor prognosis. Clin Cancer Res., 6(2), 572-577.

Kushlinskii, N. E., Babkina, I. V., Solovev, Y. N., \& Trapeznikov, N. N. (2000). Vascular endothelium growth factor and angiogenin in the serum of patients with osteosarcoma and Ewing's tumor. Bull Exp Biol Med, 130(7), 691-693. http://dx.doi.org/10.1007/BF02682107

Ladanyi, M., \& Gorlick, R. (2000). Molecular pathology and molecular pharmacology of osteosarcoma. Pediatr Pathol Mol Med, 19, 391-413. http://dx.doi.org/10.1080/15227950050193641

Longhi, A., Benassi, M. S., Molendini, L., Macchiagodena, M., Picci, P., \& Bacci, G. (2001). Osteosarcoma in blood relatives. Oncology Rep, 8, 131-136.

Luna-Ortiz, K., Villavicencio-Valencia, V., Carmona-Luna, T., Pasche, P., \& Mosqueda-Taylor, A. (2010). Osteogenic sarcoma of the maxillary region in a mexican mestizo population. J Craniofac Surg, 21(6), 1709-1714. http://dx.doi.org/10.1097/SCS.0b013e3181f3c6d4

Marina, N., Gebhardtb, M., Teotc, L., \& Gorlickd, R. (2004). Biology and therapeutic advances for pediatric osteosarcoma. The Oncologist, 9(4), 422-441. http://dx.doi.org/10.1634/theoncologist.9-4-422

Master, S. R. (2005). Diagnostic proteomics: back to basics? Clin Chem, 51, 1333-1334. http://dx.doi.org/10.1373/clinchem.2005.053686

Meng, Z., \& Veenstra, T. D. (2007). Proteomic analysis of serum, plasma and lymph for the identification of biomarkers. Proteomics Clin Appl, 1, 747-757. http://dx.doi.org/10.1002/prca.200700243

Mirbello, L., Troisi, R. J., \& Savage, S. A. (2009). International osteosarcoma incidence patterns in children and adolescents, middle ages and elderly persons. Int J Cancer, 125, 229-234. http://dx.doi.org/10.1002/ijc.24320

Orellana, C. (2005). Cancer care for mexican children. Lancet Oncol, 6(2), 70. http://dx.doi.org/10.1016/S1470-2045(05)01723-7

Ottaviani, G., \& Jaffe, N. (2009). The epidemiology of osteosarcoma. Cancer Treatment and Research, 152, 3-13. http://dx.doi.org/10.1007/978-1-4419-0284-9_2

Pepe, M. S., Etzioni, R., Potter, J. D., \& Thompson, M. L. (2001). Phases of biomarker development for early detection of cancer. J Nat Cancer Inst, 93, 1054-1061. http://dx.doi.org/10.1093/jnci/93.14.1054

Pepene, C. E., Kasperk, C. H., Pfeilschifter, J., Börcsök, I., Gozariu, L., Ziegler, R., \& Seck, T. (2001). Effects of triiodothyronine on the insulin-like growth factor system in primary human osteoblastic cells in vitro. Bone, 29(6), 540-546. http://dx.doi.org/10.1016/S8756-3282(01)00607-X

Petrosyan, A. S., Ivanova, N. M., Pashkov, Y. V., \& Dzampaev, A. Z. (2003). Prognostic factors of osteosarcoma in children. Pediatric Oncology, 2, 38-40.

Picci, P. (2007). Osteosarcoma (osteogenic sarcoma). Orphanet Journal of Rare Diseases, 2(1, article 6). http://dx.doi.org/10.1186/1750-1172-2-6

Pierz, K. A., Womer, R. B., \& Dormans, J. P. (2001). Pediatric bone tumors: osteosarcoma, Ewing’s sarcoma, and chondrosarcoma associated with multiple hereditary osteochondromatosis. J Pediat Orthopedics, 21, 412-418. http://dx.doi.org/10.1097/01241398-200105000-00028 
Raica, M. (2009). Angiogenesis in pre-malignant conditions. Eur J Cancer, 45, 1924-1934. http://dx.doi.org/10.1 016/j.ejca.2009.04.007

Rendón-Macías, M. E., Mejía-Aranguré, J. M., Juárez-Ocaña, S., \& Fajardo-Gutiérrez, A. (2005). Epidemiology of cancer in children under one year of age in Mexico City. Eur J Cancer Prev, 14(2), 85-89. http://dx.doi.org/10.1097/00008469-200504000-00003

Savitskaya, Y. A., Rico, G., Linares, L., Gonzalez, R., Tellez, R., Estrada, E., ... Ibarra, C. (2010). Circulating natural IgM antibodies against angiogenin in the peripheral blood sera of patients with osteosarcoma as candidate biomarkers and reporters of tumorigenesis. Biomarker in Cancer, 2, 65-78. http://dx.doi.org/10.4137/BIC.S6040

Sawyers, C. I. (2008). The cancer biomarker problem. Nature, 452, 548-552. http://dx.doi.org/10.1038/nature06913

Sidorenko, J. S., Kozlova, M. B., Frantsijants, E. M., Kozel, J. J., \& Sidorenko, L. J. (2004). Method of predicting process generalisation in case of malignant bone tumors. Patent RU 2427323 C1 Russian Federation.

Siegel, R., Naishadham, D., \& Jemal, A. (2012). Cancer statistics, 2012. Cancer J Clin, 62(1), 10-29. http://dx.doi.org/10.3322/caac.20138

Silha, J. V., Krsekt, M., Hanat, V., Marekt, J., Weisst, V., Jezkovat, J., ... Murphy, L. (2005). The effects of growth hormone status on circulating levels of vascular growth factors. Clin Endocrinol, 63(1), 79-85. http://dx.doi.org/10.1111/j.1365-2265.2005.02303.x

Technical Division of Statistical Information in Health. IMSS in numbers. 1990-2003. (2005). Rev Med Inst Mex Seguro Soc, 43(4), 349-56. Spanish.

Tunn, P. U., Schmidt-Peter, P., Pomraenke, D., \& Hohenberger, P. (2004). Osteosarcoma in children: long-term functional analysis. Clin Orthop, 421, 212-217. http://dx.doi.org/10.1097/01.blo.0000126301.34087.b4

Vollmers, H. P. (2007). Natural IgM antibodies and cancer. J Autoimmun, 29, 295-302. http://dx.doi.org/10.1016 /j.jaut.2007.07.013

Wang, L. L. (2005). Biology of osteogenic sarcoma. Cancer J., 11(4), 294-305. http://dx.doi.org/10.1097/00130 404-200507000-00005

Williams, G. R., Bland, R., \& Sheppard, M C. (1994). Characterization of thyroid hormone (T3) receptors in three osteosarcoma cell lines of distinct osteoblast phenotype: interactions among T3, vitamin D3, and retinoid signaling. Endocrinology, 135(6), 2375-2385. http://dx.doi.org/10.1210/en.135.6.2375

Yaw, K. M. (1999). Pediatric bone tumors. Semin Surg Oncol, 16, 173-183. http://dx.doi.org/10.1002/(SICI)109 8-2388(199903)16:2<173::AID-SSU8>3.3.CO;2-7

Zhong, L., Peng, X., Hidalgo, G. E., Doherty, D. E., Stromberg, A. J., \& Hirschowitz, E. A. (2004). Identification of circulating antibodies to tumor-associated proteins for combined use as markers of non-small cell lung cáncer. Proteomics, 4, 1216-1225. http://dx.doi.org/10.1002/pmic.200200679 